\title{
Screened AAV variants permit efficient transduction access to supporting cells and hair cells
}

\author{
Xinde $\mathrm{Hu}^{1,2}$, Jinghan Wang ${ }^{3,4}$, Xuan Yao', Qingquan Xiao ${ }^{1,2}$, Yuanyuan Xue ${ }^{3,4}$, Shaoran Wang ${ }^{1}$, Linyu Shi', Yilai Shu ${ }^{3,4}$, \\ Huawei $\mathrm{Li}^{3,4}$ and Hui Yang ${ }^{1}$
}

Dear Editor,

Genetic malady is a significant pathogenesis underlying sensorineural hearing loss. More than half of the individuals with pre-lingual hearing loss are verified with inherited genetic defects, which are becoming a severe public health issue around the world ${ }^{1}$. Despite the impressive progress in identification of genes associated with deafness, no medical treatment is available for genetic hearing loss except for cochlear implantation. Therefore, developing new treatments that target diverse types of genetic hearing loss is a high priority. Inner ear gene therapy is a promising therapeutic approach to develop treatments for genetic hearing loss. The mammalian cochlea contains two types of hair cells (HCs): inner HCs (IHCs) and outer HCs (OHCs), both of which are essential for the detection and processing of auditory signal $^{2}$. These HCs are surrounded by supporting cells (SCs), a heterogeneous group of cells that are important for cochlear homeostasis ${ }^{3}$. Moreover, SCs are closely associated with genetic defects, resulting in the most common hearing impairments ${ }^{4,5}$. However, the inefficiencies of gene introduction into HCs and SCs not only limit the study of the function of inner ear genes, but also hinder gene therapy application for hereditary deafness. A

\footnotetext{
Correspondence: Yilai Shu (yilai_shu@fudan.edu.cn) or

Huawei Li (hwli@shmu.edu.cn) or Hui Yang (huiyang@ion.ac.cn)

${ }^{1}$ Institute of Neuroscience, State Key Laboratory of Neuroscience, Key Laboratory of Primate Neurobiology, CAS Center for Excellence in Brain Science and Intelligence Technology, Shanghai Research Center for Brain Science and Brain-Inspired Intelligence, Shanghai Institutes for Biological Sciences, Chinese Academy of Sciences, Shanghai 200031, China

${ }^{2}$ College of Life Sciences, University of Chinese Academy of Sciences, Beijing 100049, China

Full list of author information is available at the end of the article. These authors contributed equally: Xinde Hu, Jinghan Wang, Xuan Yao, Qingquan Xiao
}

proper vector for efficient gene delivery into targeted HCs and SCs is a prerequisite for accurate gene therapy.

In the past decade, several gene delivery technologies, including nanoparticles, have been developed for gene delivery into the inner ear. Non-viral nanoparticles, most often made from synthetic and cationic lipid or polymer delivery materials, can be used to deliver genome-editing tools in vitro and in vivo. However, the presence of additional extracellular barriers hinders in vivo application. As a result, most of them have limited gene delivery efficiency in vivo ${ }^{6,7}$.

Adeno-associated virus (AAV) has been certified competent to achieve the safe, efficient, and endurable outcomes of gene therapy. By injection through the round window membrane (RWM), several AAV vectors in different serotypes have been shown to exhibit effective $\mathrm{IHCs}$ transduction, but difficultly in targeting the $\mathrm{OHCs}$ and SCs remains, leading to only partial correction of hearing in mouse models of inherited deafness ${ }^{8,9}$. Thus, researchers have tried to expand the tropism of viral vectors to simultaneously target cells of various subtypes, including IHCs, OHCs, and SCs. Artificial recombinant $A A V$ vectors have been created to improve infectivity and enhance their ability to avoid neutralization by preexisting antibodies in the circulatory system ${ }^{10}$. Pseudoserotype vectors, AAV-PHP.eB and AAV-DJ, have been shown to outperform various naturally occurring AAVs in different serotypes in their ability to transduce multiple tissues and organs in vitro and in vivo ${ }^{10-12}$.

In order to screen AAV vectors that target $\mathrm{HCs}$ and SCs with high efficiencies, we packaged different subtypes of AAVs (AAV-8, AAV-9, AAV-DJ, and AAV-PHP.eB for HCs; AAV-8, AAV-9, AAV-DJ for SCs) that express tdTomato and separately injected them through the 
a

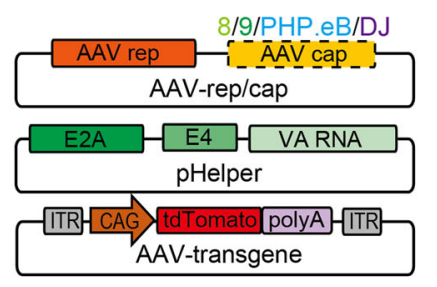

b

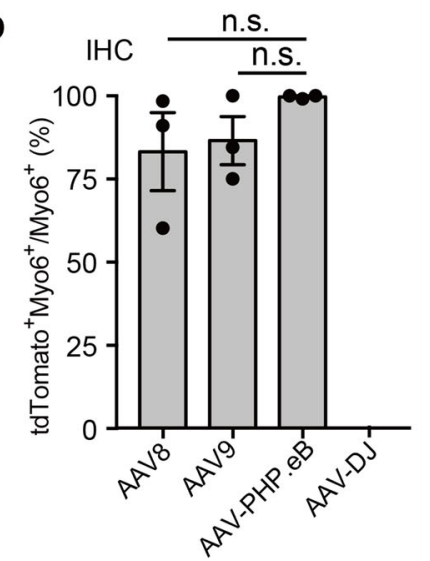

e

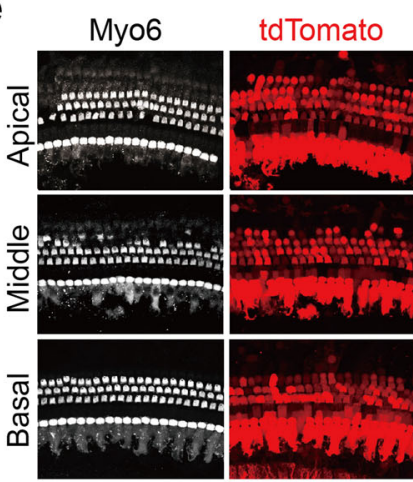

g

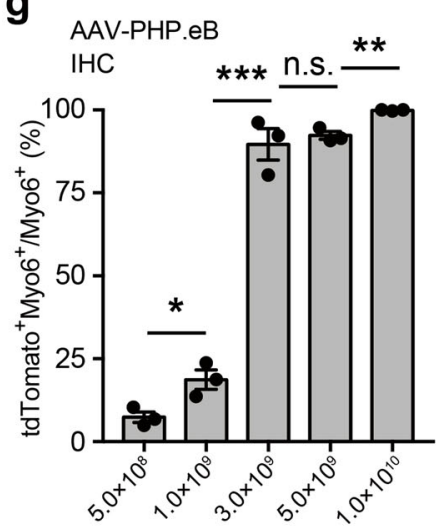

C

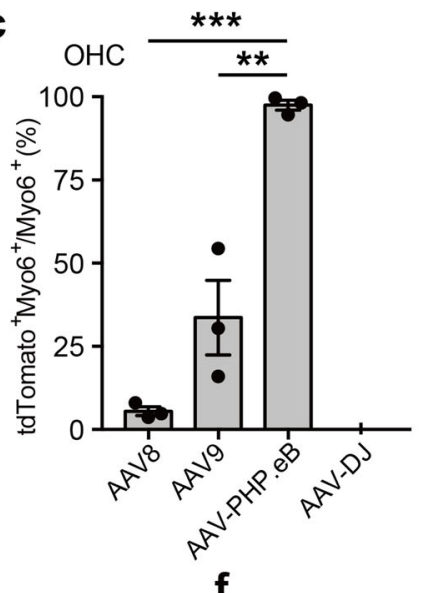

Merge

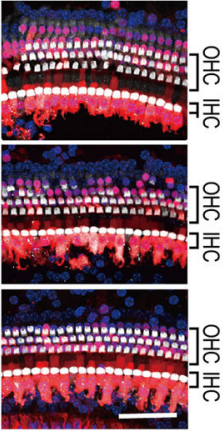

$\mathbf{h}$

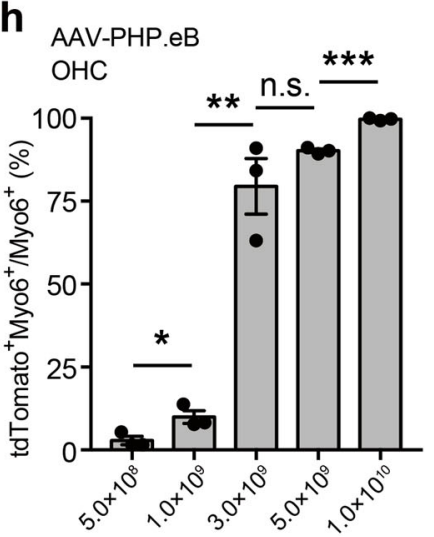

i d

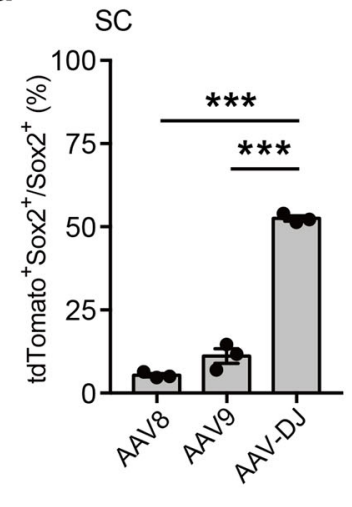

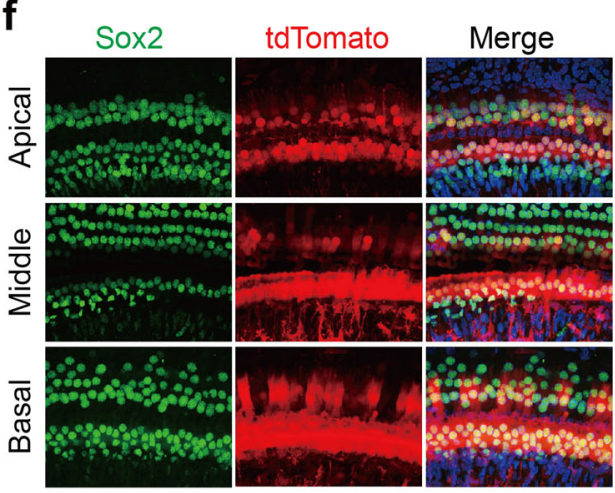

AAV-DJ

SC

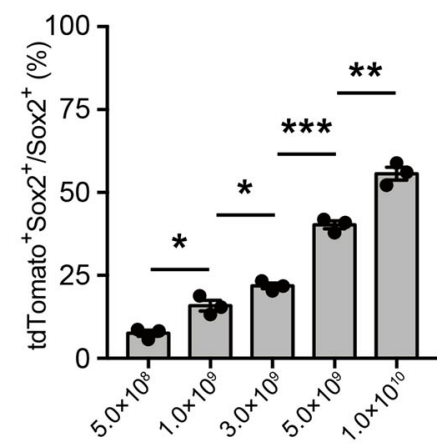

Fig. 1 Infection efficiencies of different adeno-associated virus (AAV) variants for hair cells (HCs) and supporting cells (SCs). a Schematic overview of infection screening in hair cells and supporting cells using different subtypes of AAVs. Different subtypes of AAVs (AAV-8/9/PHP.eB/DJ) were packaged and injected into the cochlea of P1 Institute of Cancer Research (ICR) mice. The injected cochlea regions were dissected for immunostaining and phenotype analysis at 3 weeks post injection. $\mathbf{b}$ - $\mathbf{d}$ Infection efficiencies of different AAV subtypes measured by the percentage of tdTomato $^{+}$cells in inner HCs $(\mathrm{IHCS})(\mathbf{b})$, outer $\mathrm{HCs}(\mathrm{OHCS})(\mathbf{c})$, and SCs (d). Results were obtained from three animals and are presented as mean \pm SEM. For each animal, $\mathrm{HC}$ and SC infection was quantified at six different locations along the cochlea: two at the apex, two at the middle turn, and two at the cochlear base. ${ }^{*} P<0.05,{ }^{*} P<0.01$, and ${ }^{* *} P<0.001$, unpaired Student's $t$ test. e, $\mathbf{f}$ Representative immunofluorescence images of HCs and SCs in cochlea sections injected with AAV-PHP.eB or AAV- DJ at 3 weeks post injection. Myo6, HC marker; Sox2, SC marker; tdTomato, transfected cells. Scale bar, 50 um. g-i Infection efficiencies of different doses of AAV-PHP.eB and AAV-DJ in IHCs (g), OHCs (h) and SCs (i). Results were obtained from three animals and are presented as mean \pm SEM. ${ }^{*} P<0.05$, ${ }^{* *} P<0.01$, and ${ }^{* *} P<0.001$; n.S., no significance; unpaired Student's $t$ test 
RWM of mice at a dose of $1 \times 10^{10}$ virus genome $(\mathrm{vg})$ (Fig. 1a). Three weeks after injection, modiolar crosssections of the cochleae showed broad distribution of tdTomato expression in all four serotypes of AAV (Supplementary Fig. S1). TdTomato expression in diverse inner ear cell types, including sensory epithelial, spiral ganglion, spiral ligaments, stria vascularis, and spiral limbus, was observed in all four AAV groups (Supplementary Fig. S1). We further immunostained the sections and found that AAV-8 and AAV-9 infected the IHCs but not the OHCs nor SCs, which was consistent with previous studies (Fig. 1b, d; Supplementary Fig. S2). However, we found that PHP.eB transduced both the IHCs and OHCs effectively (Fig. 1e). Surprisingly, we found that tdTomato expression was detected in SCs in AAV-DJ injection group (Fig. 1f; Supplementary Fig. S2).

To evaluate the infection rate of each AAV serotype in different cell types, we conducted immunofluorescence staining on whole-mounted cochleas 3 weeks after injection. We found that AAV-8, AAV-9, and AAV-PHP.eB robustly infected the IHCs, while AAV-DJ showed very rare infection (Fig. 1b, e; Supplementary Fig. S3 and Supplementary Table S1). In the apical turn, tdTomato expression was observed in $98.94 \pm 1.30 \%, 98.41 \pm 1.94 \%$, and $100.00 \pm 0.00 \% \mathrm{IHCs}$ in the AAV-8, AAV-9, and AAV-PHP.eB groups, respectively. In the middle and basal turn, AAV-PHP.eB also achieved very high infection efficiency $(99.07 \pm 1.13 \%$ and $100.00 \pm 0.00 \%$ ) (Fig. 1b, e), while AAV-8 and AAV-9 achieved relatively lower infection efficiencies $(76.83 \pm 27.41$ and $73.91 \pm 17.15 \%$ for AAV-8; $92.05 \pm 5.06$ and $69.16 \pm 20.17 \%$ for AAV-9) (Supplementary Table S1).

It is widely accepted that the OHCs are much more difficult to infect with AAV. The infection efficiencies of OHCs using AAV-8 and AAV-DJ were minimal $(<5 \%)$, and AAV-9 showed a moderate infection efficiency $(33.62 \pm 13.72 \%)$ (Fig. 1c; Supplementary Table S1). By contrast, we found AAV-PHP.eB infected OHCs with a high efficiency, with the average percentages of tdTomato-positive $\mathrm{OHCs}$ in the apical, middle, and basal turns being $98.6 \%, 96.2 \%$, and $97.6 \%$, respectively (Supplementary Table S1). Surprisingly, we found that AAVDJ showed much higher efficiency $(52.51 \pm 0.96 \%)$ for SC infection than AAV-8 or AAV-9 $(5.38 \pm 0.63$ and $11.10 \pm$ $2.70 \%$ ) (Fig. 1d, f; Supplementary Fig. S4 and Supplementary Table S1).

To further determine the infection ability of these two AAV serotypes for $\mathrm{HCs}$ and SCs, we injected different doses of AAV-PHP.eB and AAV-DJ $\left(5 \times 10^{8}\right.$ to $\left.1 \times 10^{10} \mathrm{vg}\right)$ into the neonatal mouse cochlea. We found that both IHCs and $\mathrm{OHCs}$ were infected efficiently with only $3 \times 10^{9} \mathrm{vg}$ AAV- PHP.eB (Fig. 1g, h; Supplementary Fig. S5 and Supplementary Table S2); the required AAV dosage was much lower than previously reported serotypes ${ }^{13}$. In addition, we found that AAV-DJ infected up to $50 \%$ of SCs with a dose of $1 \times 10^{10} \mathrm{vg}$ (Fig. 1i; Supplementary Fig. S6 and Supplementary Table S2).

To determine the safety of AAV-DJ and PHP.eB for gene transfer, we assessed whether the delivery of AAVs had any effect on normal auditory function using the auditory brainstem response threshold test. At all the measured frequencies, we did not observe any difference between the AAV-PHP.eB- or AAV-DJ-injected ears and the contralateral control ears (Supplementary Fig. S7). Therefore, AAV-DJ and PHP.eB demonstrated no obvious toxicity and could be potential AAV vectors for inner ear research and gene therapy.

In summary, we found that different recombinant AAVs exhibited distinctive traits in targeting tropism, and two subtypes of AAVs could achieve high efficiencies of infection in HCs and SCs. AAV-PHP.eB showed extremely high transduction efficacy on both OHCs and IHCs, even at incremental diluted scales. Although several recent studies have validated some new AAV vectors in mouse inner ear HC infection, AAV-PHP.eB is the most efficient AAV vector for gene delivery observed to date. Using high titer AAVs, AAV2.7m8, Anc80L65, and PHP.B can infect about $85 \%, 65 \%$, and $50 \%$ of OHCs, respectively, while AAV-PHP.eB infect nearly $100 \%$ of the OHCs at a relatively low dose ${ }^{13,14}$. The infection rate difference of OHCs may due to the affinity of different AAV serotypes. We also demonstrated that AAV-DJ had a relatively high infection efficiency in SCs, surpassing what has been reported previously ${ }^{15}$. Overall, these findings suggest that AAV-PHP.eB and AAV-DJ hold great promise for gene delivery into inner ear, for both basic research and gene therapy purposes.

\footnotetext{
Acknowledgements

This work was supported by R\&D Program of China (2018YFC2000100 and 2017YFC1001302), CAS Strategic Priority Research Program (XDB32060000), National Natural Science Foundation of China (31871502 (to H.Y.); 81771013 , 81822011 (to Y.S.) and 81620108005 (to H.L.)), Shanghai Municipal Science and Technology Major Project (2018SHZDZX05), Shanghai City Committee of science and technology project (18411953700, 18JC1410100, 16JC140202), and Science and Technology Commission of Shanghai Municipality (17ZR1448600 and 18410712400 to Y.S.).
}

\section{Author details}

'Institute of Neuroscience, State Key Laboratory of Neuroscience, Key Laboratory of Primate Neurobiology, CAS Center for Excellence in Brain Science and Intelligence Technology, Shanghai Research Center for Brain Science and Brain-Inspired Intelligence, Shanghai Institutes for Biological Sciences, Chinese Academy of Sciences, Shanghai 200031, China. ${ }^{2}$ College of Life Sciences, University of Chinese Academy of Sciences, Beijing 100049, China. ${ }^{3}$ ENT Institute and Otorhinolaryngology Department, Affiliated Eye and ENT Hospital, State Key Laboratory of Medical Neurobiology, Fudan University, Shanghai, China. ${ }^{4} \mathrm{NHC}$ Key Laboratory of Hearing Medicine (Fudan University), Shanghai 200031, China

\section{Author contributions}

H.Y., H.L., and Y.S. conceived the project and designed experiments. X.H. and J.W. performed injection and analyzed the data. Q.X., Y.X., and S.W. performed 
tissue slicing and immunostaining. L.S. performed AAV package. X.H., X.Y., and H.Y. wrote the paper.

\section{Conflict of interest}

The authors declare that they have no conflict of interest.

\section{Publisher's note}

Springer Nature remains neutral with regard to jurisdictional claims in published maps and institutional affiliations.

Supplementary Information accompanies the paper at (https://doi.org/ 10.1038/s41421-019-0115-9).

Received: 3 March 2019 Accepted: 19 July 2019

Published online: 15 October 2019

\section{References}

1. Rennels, M. \& Pickering, L. K. Sensorineural hearing loss in children. Lancet 365, 2085-2086 (2005).

2. LeMasurier, M. \& Gillespie, P. G. Hair-cell mechanotransduction and cochlear amplification. Neuron 48, 403-415 (2005).

3. Salt, A. N. \& Hirose, K. Communication pathways to and from the inner ear and their contributions to drug delivery. Hear. Res. 362, 25-37 (2018).

4. Geleoc, G. S. \& Holt, J. R. Sound strategies for hearing restoration. Science $\mathbf{3 4 4}$ 1241062 (2014).
5. Yu, Q. et al. Virally expressed connexin26 restores gap junction function in the cochlea of conditional Gjb2 knockout mice. Gene Ther. 21, 71-80 (2014).

6. Cronican, J. J. et al. Potent delivery of functional proteins into mammalian cells in vitro and in vivo using a supercharged protein. Acs Chem. Biol. 5, 747-752 (2010).

7. Hao, J. \& Li, S. K. Inner ear drug delivery: recent advances, challenges, and perspective. Eur. J. Pharm. Sci. 126, 82-92 (2019).

8. Askew, C. et al. Tmc gene therapy restores auditory function in deaf mice. Sci. Transl. Med. 7, https://doi.org/10.1126/scitranslmed.aab1996 (2015).

9. Shu, Y. et al. Identification of adeno-associated viral vectors that target neonatal and adult mammalian inner ear cell subtypes. Hum. Gene Ther. 27, 687-699 (2016).

10. Grimm, D. et al. In vitro and in vivo gene therapy vector evolution via multispecies interbreeding and retargeting of adeno-associated viruses. J. Virol. 82, 5887-5911 (2008)

11. Chan, K. Y. et al. Engineered AAVs for efficient noninvasive gene delivery to the central and peripheral nervous systems. Nat. Neurosci. 20, 1172 (2017).

12. Deverman, B. E. et al. Cre-dependent selection yields AAV variants for widespread gene transfer to the adult brain. Nat. Biotechnol. 34, 204 (2016).

13. Isgrig, K. et al. AAV2.7m8 is a powerful viral vector for inner ear gene therapy. Nat. Commun. 10, 427 (2019).

14. Gyorgy, B. et al. Gene transfer with AAV9-PHP.B rescues hearing in a mouse model of Usher Syndrome $3 \mathrm{~A}$ and transduces hair cells in a non-human primate. Mol. Ther. 13, 1-13 (2019).

15. Kim, M. A. et al. Targeted gene delivery into the mammalian inner ear using synthetic serotypes of adeno-associated virus vectors. Mol. Ther. 13, 197-204 (2019). 